was identified as the single greatest enabler of return to work. Although pockets of good practice were identified, in the main, health professionals and employers describe uncertainty about their role and responsibilities and describe a narrow scope of practice. Patients report a mostly adversarial experience of vocational supports.

Conclusions: Irish vocational rehabilitation stakeholders do not report awareness of the complex interplay of biological, psychological and social factors influencing work participation for people with MSD's. Vocational supports and services are hampered by role uncertainty and consequentially adversarial experiences for service users.

Acknowledgements: The authors acknowledge funding support from the Health Research Institute, Univeristy of Limerick for this study.

Disclosure of Interest: None declared

DOI: 10.1136/annrheumdis-2018-eular.1432

\section{AB1459-HPR PATIENTS WITH RHEUMATOID ARTHRITIS HAVE LATERALITY ON THE UPPER LIMBS RANGE OF MOTION}

K Tsujimura ${ }^{1}$, S. Kato ${ }^{1}$, C. Nagata ${ }^{1}$, S. Nakazaki ${ }^{2} .{ }^{1}$ Rheumatology, Kamiaraya Clinic; ${ }^{2}$ Rheumatology, Johoku Hospital, kanazawa, Japan

Background: Biological disease-modifying antirheumatic drug (bDMARD) has been introduced since 2003 in Japan. In many patients, bDMARD has made it possible to control the disease activity of rheumatoid arthritis (RA), and has led to the structural remission and the functional remission. As for the structural damage, the relationship between mechanical stress and radiographic damage in RA has been recently reported. Koh reported that radiographic damage was worse and progressed more rapidly in the dominant hand in individuals with early RA ${ }^{1}$. Nakazaki reported that the eroded joint count was significantly more in the dominant than the non-dominant upper extremity ${ }^{2}$. Incidentally, there are few studies as for the relationship between mechanical stress and functional impairment, while joint range of motion (ROM) plays a important role on the physical function of the patients with RA.

Objectives: The aim of this study is to examine whether patients with RA have difference between right and left on the upper limbs and to see whether mechanical stress influences on ROMs.

Methods: We assessed 103 RA female patients aged less than 75 years old with their disease onsets after 2003 and their disease durations within 10 years. Exclusion criteria were the patients with past history of any apoplexy or fracture. We measured the ROM of joints including the shoulder, elbow, wrist, hip, knee, and ankle. The ROMs were measured by criteria of the Japanese Orthopaedic Association and the Japanese Association of Rehabilitation Medicine, and then the joints with restricted ROM were counted to see if any difference between right and left limbs. Data were analysed by using McNemar's test and judged as statistically significant when $p$ value was less than 0.01

Results: The mean age was 57.1 (26 74) years, the mean disease duration was 64.0 (7 120) months, the rate of bDMARD was $33.3 \%$, the right limb was dominant in $99.0 \%$ among the patients. As for the ROM of upper limbs, the forearm pronation and the wrist extension of the right limbs demonstrated significantly more restricted ROM compared with the left limbs $(p<0.001)$. The ROM of lower limbs showed no significant laterality.

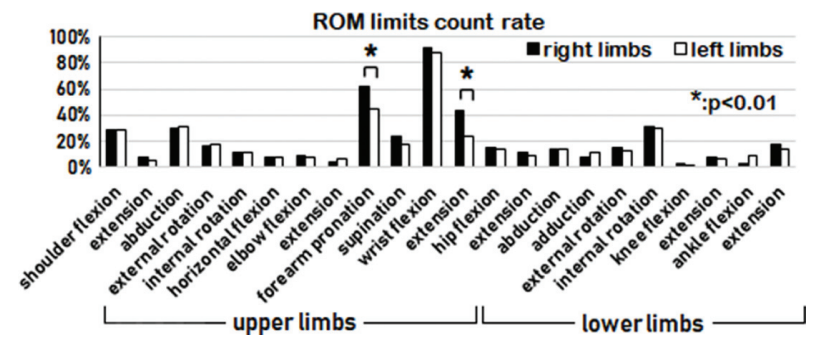

Abstract AB1459-HPR - Figure 1

Conclusions: The ROM limit count in the upper limbs was significantly more in the right limbs than the left limbs and the right limb was dominant in $99 \%$ of the patients, therefore it was suggested that the mechanical stress influenced the ROM of upper limbs and physical function in patients with RA even in the era of the bDMARD.
REFERENCES:

[1] Jung Hee Koh, et al.: Radiographic structural damage is worse in the dominant than the non-dominant hand in individuals with early rheumatoid arthritis. PLOS ONE, doi: 10. 1371/journal. Phone.0135409 August 6, 2015

[2] Satoshi Nakazaki: Radiographic structural damage is worse in the dominant than the non-dominant upper extremity in patients with rheumatoid arthritis. poster from eular, June 2017

Disclosure of Interest: None declared

DOI: 10.1136/annrheumdis-2018-eular.1641

\section{AB1460-HPR PSYCHOLOGICAL VARIABLES PREDICTOR OF SLEEP DISORDERS IN PATIENTS WITH ANKILOSING SPONDILYLITIS AND PSORIASTIC ARTRTITS. MULTICENTER STUDY}

L Cano Garcia ${ }^{1}$, N. Mena-Vázquez ${ }^{1}$, M.D. Hernández-Sánchez ${ }^{2}$, R. SEGURARUIZ $^{3}$, C. Domínguez-Quesada ${ }^{4}$, S. Manrique-Arija ${ }^{1}$, I. Ureña-Garnica ${ }^{1}, F$. G. Jiménez-Núñez ${ }^{1}$, E. Vilchez-Ocaña ${ }^{1}$, A. Fernández-Nebro ${ }^{5} .{ }^{1}$ Rheumatology, Hospital Regional Universitario De Málaga, Instituto De Investigación Biomédica De Málaga(ibima), Universidad De Málaga, Málaga; ${ }^{2}$ Rheumatology, Complejo Hospitalario de Jaén, Jaén; ${ }^{3}$ Rheumatology, Hospital Reina Sofía, Córdoba; ${ }^{4}$ Rheumatology, Hospital Virgen Macarena, Sevilla; ${ }^{5}$ Rheumatology, Hospital Regional Universitario De Málaga, Instituto De Investigación Biomédica De Málaga (ibima), Departamento De Medicina Y Dermatología, Universidad De Málaga, Málaga, Spain

Objectives: To study the variables associated with the severity of insomnia and hypersomnia according to the Oviedo sleep questionnaire (COS) in patients with ankylosing spondylitis (AS) and psoriatic arthritis (PSA). Methods: Design. Transversal descriptive study. PATIENTS: Patients with follow-up AS or PSA were selected by consecutive sampling in rheumatology units of 4 Spanish cities. Selection criteria: age $\geq 16$ years old) with AE (New York criteria) or PSA (CASPAR criteria) able to understand and willing to carry out the questionnaires. Protocol: they were explained and the patients were given to meet the selection criteria and the informed consent signed a battery of questionnaires; his doctor performed the evaluation of disease activity and recorded comorbidities and current medication. Main outcomes: the 3 dimensions of $\mathrm{COS}:{ }^{1}$ Subjective satisfaction with sleep, ${ }^{2}$ Insomnia and ${ }^{3}$ Hyperinsomnia. Other variables: current medication for $\mathrm{AE}$ or $\mathrm{PsA}$, comorbidities, use of sleeping drugs and/or CPAP according to the COS questionnaire, disease activity: axial AS (BASDAI) and PSA (DAS28), quality of life related to health (HRQOL) through SF-36, pain perception (Brief Pain Inventory BPI questionnaire), AE (BASDAI) and PSA (DAS28) and fatigue through FACIT, emotional intelligence through TMMS, resilience with the resilience questionnaire, anxiety screening and depression through HADs. Statistical analysis: Descriptive analysis of the main variables. Bivariate using T-Student, Mann Whitney $U$ and $\chi 2$ followed by binary logistic regression (RLB) (Vd: Insomnia: value greater than 22 of the insomnia scale).

Results: 302 patients participated: 152 patients with $A E$ and 149 patients with PA $(47.8 \%$ women, $48.97 \pm 10.26$ years) with an average of $8.35 \pm 6.8$ years of disease. They used $47.8 \%$ biological therapy. The most frequent comorbidities were: disc disease 143 (47.5\%), visual impairment 60 (19.9\%), anxiety 49 (16.3\%), obesity $47(15.6 \%)$ and depression 45 $(15 \%)$.In the bivariate analysis it was observed that patients with $A E$ suffered more insomnia than patients with PSA $(57 \%$ vs $43 \%, p=0.029)$, they took more drugs to sleep $(30.3 \%$ vs $22.9 \%, p=0.001)$ and suffered a higher activity of the disease measured by BASDAI (mean [SD] 4.7 [1.8] vs 3.8 [1.9], $p=0.005)$. In insomnia in PsA, it was not associated with the degree of activity measured by DAS28 but it was associated with depression. In the binary logistic regression analysis (RLB), insomnia was associated with BASDAI and depression. Therefore, patients with greater BASDAI and depression have almost twice the risk of presenting insomnia in patients with spondyloarthropathies. There are no associations of insomnia with the resilience and emotional intelligence with results above the cut, that is, patients with good resilience and emotional intelligence.

Conclusions: There is an association between insomnia, disease activity and depression in the group of $A E$, in the case of PSA there is a relationship with depression. Insomnia is a problem related to depression, so education in sleep hygiene in nursing consultation could be of help for insomnia.

Disclosure of Interest: None declared

DOI: 10.1136/annrheumdis-2018-eular.4303 